\title{
MONTE CARLO METHODS IN THE PHYSICAL SCIENCES
}

\author{
Malvin H. Kalos \\ Lawrence Livermore National Laboratory \\ Livermore, CA 94551, USA
}

\begin{abstract}
I will review the role that Monte Carlo methods play in the physical sciences. They are very widely used for a number of reasons: they permit the rapid and faithful transformation of a natural or model stochastic process into a computer code. They are powerful numerical methods for treating the many-dimensional problems that derive from important physical systems. Finally, many of the methods naturally permit the use of modern parallel computers in efficient ways. In the presentation, I will emphasize four aspects of the computations: whether the computation derives from a natural or model stochastic process; whether the system under study is highly idealized or realistic; whether the Monte Carlo methodology is straightforward or mathematically sophisticated; and finally, the scientific role of the computation.
\end{abstract}

\section{INTRODUCTION}

Over the last four decades, numerical computation has played an increasingly important role in both theoretical and experimental science. The reasons are not hard to understand: the increasing sophistication of our understanding of physical phenomena coupled with our increasing need to create ever more subtle processes and devices requires a level of analysis and prediction beyond that of traditional mathematical methods. When one sets out to design a modern high-energy accelerator, any of its enormous detectors, a proposed experiment, or to analyze the results, computation must be invoked at every stage of the process. Inference from most astronomical observations is usually a matter of significant computer-aided analysis. A complementary situation exists in theoretical science. Serious quantitative prediction of a chemical reaction rate, the behavior of a nuclear reactor, or the energy of an atomic nucleus from more fundamental information requires computation, often very intensive.

Monte Carlo methods play a central role in many of these computations. For example, the prediction of behavior of a high-energy particle detector is most easily and most accurately analyzed by a Monte Carlo treatment that simulates the stochastic processes of the creation of parti- cles in a target, the decay of those particles into others, the transport of particles, and their final interactions with the detection process. Such a calculation is formidable but straightforward on modern computers. Because the computation deals with a succession of independent histories, it is easily parallelized as well.

It is also worth mentioning that the full description of the state of a particle decay process in a particle detector requires many dimensions - there may be a number of particles simultaneously present in the system, and for each a position, time, and momentum must be specified- a total of seven dimensions for each particle. An important theme of the talk is that Monte Carlo methods offer a natural and efficient procedure for numerical problems in many dimensions that are impossible by more traditional numerical methods.

The kinetic theory of gases - that is the description of their behavior from that of constituent atoms or molecules - is one of the longest-standing examples of statistical physics, dating to Daniel Bernoulli in 1738, with important progress by John Herapath in 1820, James Maxwell in 1859, and A. Einstein in 1905. It is a natural candidate for Monte Carlo, and implementations were pioneered by Bird (1994).

Kinetic Monte Carlo starts with a stochastic model of atomistic processes in a dense material, say a solid. Simple examples are defined on a lattice in two or three dimensions, and assume that some of the lattice points are occupied by one or more species of atoms. Rates are specified for the addition, deletion, migration, or interaction of atoms, and a simulated stochastic process is followed on a computer. We will discuss one example in greater detail in the talk, but this is a rich area of research: The model can be highly simplified to generate insight about equilibrium and non-equilibrium statistical physics and chemistry, or it can aim for significant physical realism in support of the annealing of epitaxial growth, to cite one example of an industrially important process.

The applications of Monte Carlo methods extend far beyond the simulations of natural stochastic processes. Broadly speaking, they are used for the evaluation of definite integrals or integrals derived from the solution of an 
integral equation - that is of the density of random walkers obeying some abstract stochastic process.

One example of this is the evaluation of the equation of state of hard discs in two dimensions or hard spheres in three. What is required is the average of some property of the positions of discs or spheres over all possible nonoverlapping configurations. For an N-body system, this is a $2 \mathrm{~N}$ - or $3 \mathrm{~N}$ - dimensional integral, well suited in principle for Monte Carlo treatment. The technical challenge here is to sample the distribution of positions in an unbiased way. It was to treat this problem that the algorithm variously called the "Metropolis" or "Markov chain" or M(RT)2 was invented. It is an enormously versatile and powerful method whose generalization has led to widespread developments in the applications of Monte Carlo. The problem of the statistical mechanics of hard discs or spheres is, of course, a highly simplified physical system, but the insight it brings to more realistic models is invaluable. In addition, the method can - and has been - applied directly to systems of ever growing complexity.

Another broad application that does not arise from a physically defined stochastic process is that of integrating the non-relativistic Schrödinger equation for an N-body system. This is a description of the fundamental physics and chemistry of ordinary matter in the form of a partial differential equation in a $3 \mathrm{~N}$-dimensional space plus time. A curious fact noted early in the history of quantum mechanics is that if the physical time variable is made imaginary, then the equation is a linear diffusion equation for a walker in a $3 \mathrm{~N}$-dimension space with a non-physical but readily simulated process of creation and annihilation of walkers.

Straightforward translation of this abstraction to a simulated random walk on a computer is inefficient, and grows increasingly so as $\mathrm{N}$ grows large. But the mathematical transformation to an efficient form is not difficult and lends itself to effective computation. Indeed for some realistic physical quantum many-body systems, a numerical solution without uncontrolled approximations is possible. This will be discussed in some more detail below.

A useful recent survey of Monte Carlo methods in the physical sciences is the Proceedings of the conference held in 2003 at Los Alamos to celebrate the fiftieth anniversary of the "Metropolis Algorithm" (Gubernatis 2003).

\section{PARTICLE TRANSPORT}

Challenges in computing the transport of particles and radiation at Los Alamos during World War II led to the serious study of Monte Carlo methods by Ulam, von Neumann, Fermi, and others. The interaction of particles such as neutrons or photons with matter is, of course, a natural stochastic process, but Ulam in particular recognized that it was also the basis of efficient computational methods for many problems.
In addition, the attention by the mathematicians led to significant ideas about broad ranges of applications and about variance reduction. The theory of zero-variance Monte Carlo for linear transport was formulated in the late 1940s and has been the guide for efficient particle computations ever since (Kalos and Whitlock 1986).

Briefly, one needs approximations to the "adjoint solution", effectively the description of the stochastic process run backwards in time. This is, of course, no easier to calculate than the "forward" solution, but qualitatively reasonable approximations are often easy to establish and very efficient in practice. A widely used general purpose transport Monte Carlo program, MCNP (Monte Carlo NParticle code: MCNP 2007) from Los Alamos, includes an option for the automatic generation of adjoint information from a deterministic approximation to the physical system (Sweezy et al 2005).

\section{KINETIC THEORY OF GASES}

Contemporary very large computers have permitted atomistic calculations of gases with billions of particles. The method is widely used in the study of dilute gases, the atmosphere at high altitudes, and in general where atomistic effects in fluids need to be elucidated. An interesting and important application has been the study of the early development of instabilities in discontinuous fluids. I will show results of such calculations (Kadau et at 2007) that demonstrate agreement between theory and experimental measurements of instability growth rates. What is significant here is that the fluctuations on the atomic level - not described by the classical Navier-Stokes equation-- decisively influence the rates.

\section{KINETIC MONTE CARLO (KMC)}

There are no effective computational methods to model the dynamic behavior of many-body systems taking into account their quantum character. Studies of the evolution of the structure of condensed phases like crystals use approximation - often very good approximations - based on classical physics and on models of the potential energy functions or energetics of structural changes. Molecular dynamics (MD) - the numerical solution of Newton's equations of motion - are a widely used methodology. But the computation of atomic trajectories by MD is necessarily limited to a relatively small number of atoms for currently available computers and short times (several nanoseconds). Most crystal growth systems involve time scales upward of minutes and crystal sizes larger than microns.

As described above, $\mathrm{kMC}$ is the realization of a class of diffusive models of atomistic behavior in condensed matter, especially in solids. Rates for different processes, including the migration of atoms, are specified. Often 
these are assumed to depend upon the temperature of the system, $\mathrm{T}$, proportional to $\exp (-\mathrm{K} / \mathrm{T})$, where $\mathrm{K}$ is an activation temperature. $\mathrm{kMC}$ models based on atomic motion between discrete lattice sites have provided valuable simulations for elucidating crystal growth processes involving larger length and time scales. Atomic scale nucleation of new layers on close-packed surfaces, atomic-level step motion, surface roughening, and morphological instabilities in growing surfaces have been simulated in detail.

The straightforward translation of such models is as a time-driven simulation, but it was noted (Bortz, Kalos, and Lebowitz 1975) that an event-driven simulation would be more efficient in many applications, and this method is now used very widely. Recently, still more sophisticated methodology - first-passage algorithms - have been shown to provide speed-ups of orders of magnitude for dilute systems (Oppelstrup, et al 2006).

Early kMC simulations were the first to identify a surface roughening phase transition. (Gilmer and Bennema 1972). Crystals grown below some temperature, $T_{r}$, exhibit facets, whereas those grown at higher temperatures are rounded. This work has stimulated a large body of research on the nature of the transition and the detailed morphologies for crystals grown in the vicinity of $T_{r}$. The models have been extended to more complex systems, including the growth of polycrystalline thin films (Huang, Gilmer, and de la Rubia 1998). Systems with grain boundaries show a tendency for the formation of voids during growth of thin films from the vapor. An important aspect of thin film growth on foreign substrates is the formation of texture; that is, the development of a preferred orientation of the grains of a polycrystalline film. Simulations using kMC have shown that texture results from anisotropic growth rates. These examples illustrate the usefulness of the kMC model because of its ability to treat the atomic level phenomena that control faceting and grain boundary properties, and still allow freedom to encompass aspects such as polycrystalline structures and void formation.

$\mathrm{kMC}$ is is also used extensively for the study of irradiated materials. Energetic particles such as fission fragments or accelerated ions can cause considerable damage to crystalline materials.

In reactor materials, the diffusion of the point defects produced by decay fragments can cause problems with the stability of structural steel, and also affect the fuel rods (2). Because the lifetime of a reactor is several decades, $\mathrm{kMC}$ models can provide predictions of the evolution of the fuel rods, and guide the development of structures that will extend the operational lifetimes. (Caturla, et al 2006)

Implantation may also be useful. Energetic beams of dopant atoms are used to make $\mathrm{P}$ and $\mathrm{N}$-type regions during the fabrication of silicon devices. $\mathrm{kMC}$ models predict the final distribution of dopant atoms, and can be used to optimize that distribution by modifying the processing steps. Simulations of the paths of the incoming ions and the recoils of silicon atoms are combined with KMC diffusion models to provide an atomistic level of detail for use in predicting the final distribution of dopant atoms (Law, Gilmer, and Jaraíz 2000).

I will show a video of the evolution of a process in which aluminum atoms are deposited on an aluminum substrate at various temperatures. This is the work of Gilmer (2007). The computations were confirmed by experiments. Simulations of this kind, including many with realistic detail of nanoscale features, are widely used for quality assurance in fabrication in the electronics industry.

\section{CLASSICAL STATISTICAL MECHANICS}

Although its inventors (Metropolis et al 1953) did not follow up on the M(RT)2 method, and although theoretical scientists at the time disdained the use of computation, it proved to have a powerful impact on research on statistical mechanics in physics, chemistry, and biology. The basic idea is elegantly simple: A probability distribution, $p(\mathbf{X})$, is to be sampled for variates $\mathbf{X}$ in a many-dimensional space. A random walk is established whose dynamics are the following: A move to position $\mathbf{Y}$ is generated from the pdf $\mathrm{T}(\mathbf{Y} \mid \mathbf{X})$ and accepted with probability $\max [1$, $\mathrm{p}(\mathbf{Y}) \mathrm{T}(\mathbf{Y} \mid \mathbf{X}) / \mathrm{p}(\mathbf{X}) \mathrm{T}(\mathbf{X} \mid \mathbf{Y})]$. Under very broad conditions, the asymptotic density is $\mathrm{p}(\mathbf{X})$. In classical statistical mechanics, the function $\mathrm{p}(\mathbf{X})$ to be sampled is the Boltzmann distribution, $\exp \left[-\mathrm{V}\left(\mathrm{r}_{1}, \mathrm{r}_{2}, \ldots, \mathrm{r}_{\mathrm{N}}\right)\right]$, where $\mathrm{V}$ is the potential energy function of the coordinates $r_{k}$.

From a methodological point of view these applications have provided a wealth of generalizations, such as the observation that $\mathrm{T}(\mathbf{Y} \mid \mathbf{X})$ does not have to be a symmetric distribution.

The method has been applied to liquids, alloys, magnetic systems, and polymers, yielding accurate numerical results for, among other parameters, critical temperatures and critical exponents. For a review, see the article by Landau (2003) and the book by Landau and Binder (2000) and references therein.

\section{QUANTUM MONTE CARLO}

\subsection{Variational Quantum Monte Carlo}

Important progress in the study of many-body quantum systems was made by McMillan (1965) who observed that the function to be sampled in M(RT)2 need not be a classical Boltzmann distribution. In variational calculations a trial wave function, $\psi_{\mathrm{T}}\left(\mathrm{r}_{1}, \mathrm{r}_{2}, \ldots, \mathrm{r}_{\mathrm{N}}\right)$, is assumed, and the following quotient of integrals is to be computed: 
$E_{V}=\frac{\int \psi_{T}\left(r_{1}, r_{2}, \ldots, r_{N}\right) H \psi_{T}\left(r_{1}, r_{2}, \ldots, r_{N}\right) d r_{1} \ldots d r_{N}}{\int \psi_{T}^{2}\left(r_{1}, r_{2}, \ldots, r_{N}\right) d r_{1} \ldots d r_{N}}$

where $H=-\frac{1}{2} \nabla^{2}+V\left(r_{1}, \ldots, r_{N}\right)$ is the Hamiltonian operator. By setting the pdf $\mathrm{p}(\mathbf{X})$ in $\mathrm{M}(\mathrm{RT}) 2$ to be $\psi^{2}$, he showed that the ratio could easily be computed. Before this, expectations using even the simplest plausible many-body trial function, a "Jastrow product"

$$
\psi_{J}\left(r_{1}, \ldots, r_{N}\right)=\prod_{i<j} f\left(\left|r_{i}-r_{j}\right|\right),
$$

could only be computed by making uncontrolled approximations. The combination of unknown approximation of variational energy with unknown overestimate of the variational energy itself led to significant uncertainty in the validity of the theory.

Variational Monte Carlo is still widely used, but usually as an adjunct to the more accurate methods discussed below.

\subsection{Sign Problems}

In a number of applications one seeks solutions that are not everywhere positive. This is particularly true of "fermionic" systems, such as many-electron systems, for which the exclusion principle (Pauli 1927) demands that the wavefunction be antisymmetric, which is to say that it transforms into its negative when the coordinates of likespin electrons are interchanged. It must be negative as well as positive. Another, even greater challenge arises in treating quantum systems in physical rather than imaginary time. Here the propagators are complex so that treatment by random walk is necessarily challenging.

Some of these sign problems can be dealt with in straightforward technical ways, say by correlating negative and positive estimators, but others present deep challenges to the methodology. I will touch on some of these below.

\subsection{Green's Function Monte Carlo}

The treatment of many-particle quantum systems by simulating the non-physical diffusion of a many-dimensional random walker in imaginary time is now well developed, and applied to many systems (Kalos 1962; Kalos, Levesque, and Verlet 1974; Anderson 1976). For "bosonic" systems - like a collection of ${ }^{4} \mathrm{He}$ atoms at zero temperature, it provides a numerical method with no uncontrolled approximations (Schmidt et al 2005). The key to efficient sampling is an importance sampling transformation, namely recasting the random walk so that the density of walkers is biased by a trial function $\psi_{T}$, and modifying the transition probabilities accordingly.
Unfortunately, many important applications involve fermions, and various fixes, usually approximate, have been applied. The most usual for many-electron systems is the "fixed-node" approximation. Here, an antisymmetric trial function is used, one that has nodes, i.e., subspaces of lower dimension on which they vanish. It can be shown that if the random walk is terminated when it reaches such a point, then the energy calculated for the system is an upper bound. This is widely used, and after at least a decade of development and optimization, very good results have been attained. Research into methods that are potentially as reliable as for bosonic systems continues (Kalos and Pederiva 2000).

I will show striking results of calculations of this kind: for the homogeneous electron gas, by Ceperley and Alder (1980); for the low-lying states of light nuclei, from the work of Pandharipande and collaborators (Pandharipande 2003); and for some molecular dimers by Umrigar and Toulouse (2007).

The results for the electron gas are important for two reasons: they were the first highly reliable data for the equation of state of a many-electron system, and they form the basis of a very-widely used approximation method, "density-functional theory."

The calculations on the light nuclei are noteworthy in that the particle interactions here involve extremely complex nuclear forces. There are two-body forces derived from the study of nucleon-nucleon scattering, and which depend in complicated ways on the particles and their relative states. There are also three-body forces derived in a phenomenological way from the interactions of pions with nuclei. This means that a trial function for $\mathrm{A}=8$ comprises 17920 complex functions. Finally, the quality of the agreement with experiment validates our understanding of the structure of nuclei at this level.

The last example is that of the energies of first-row dimers (i.e., $\mathrm{Li}_{2}$ through $\mathrm{F}_{2}$ ) as calculated by quantum Monte Carlo methods, including the fixed-node approximation. To appreciate the nature of this achievement one must first understand that the energy differences that are important for chemistry are of the order of $10^{-5}$ or less of the total energy of the system. To attain this precision in a Monte Carlo calculation is evidence of sophisticated methodology. The second point is that the accuracy is very high, comparable or better than the "traditional" methods based on very large basis sets, an indication of the quality of the trial-function optimization now possible.

\subsection{Quantum Chromodynamics}

Quantum chromodynamics (QCD) is the theory of the strong force - that is, of how quarks interact via gluons to form heavy particles such as protons and neutrons (Quigg 1983). It is a field theory - that is, like quantum electrodynamics, the particles interact by way of fields that per- 
meate space. Unlike electrodynamics where the intermediating particle is a photon, both the particles (quarks) and the quanta of the field (gluons) have many possible states. There are six types of quarks and eight gluons and the interactions of quarks and gluons and gluons with themselves are mediated by matrices that couple these different states. The coupling is strong so that the kind of perturbation theory that works well for electrodynamics does not converge in QCD. Fortunately, a discretized version of the theory - lattice QCD — was formulated by Wilson (1974) which permits a numerical treatment.

A four-dimensional lattice is set up and values of the quark fields are associated with the points. The gluon interactions are represented by the links. Here too, the computed result can be expressed as an average with respect to a weighting function. The quarks are fermions so that the weighting function is not positive, but reasonable approximation schemes can make it so.

In either case, the numerical problem, for large enough lattices to approximate to a continuum, requires a Monte Carlo treatment. Indeed, even after three decades of ingenious algorithmic development and more than $10^{5}$ increase in computer speed, the lattices sizes attainable are still not quite large enough.

Nevertheless, significant predictions are now available. I will show results of lattice QCD calculations for the masses and other parameters of particles, including nucleons.

\section{CONCLUSIONS}

This rapid survey was intended to demonstrate the vast range of applicability of Monte Carlo methods to physical science, along with their great diversity of styles and subject matter. Our understanding of these powerful methods has contributed enormously to the growth of our scientific understanding. They are a significant reason for the integration of computation into modern science.

\section{ACKNOWLEDGEMENTS}

I am indebted to Cyrus Umrigar, Steven Pieper, George Gilmer, and Robert Sugar for unpublished data. This work was performed under the auspices of the U. S. Department of Energy by the University of California Lawrence Livermore National Laboratory under Contract No. W-7405Eng-48.

\section{REFERENCES}

Anderson, J. B. 1976. Journal of Chemical Physics. $65: 4122-4127$

Bird, G. A. 1994. Molecular Gas Dynamics and the Direct Simulation of Gas Flows : Clarendon
Bortz, A. B., M. H. Kalos, and J. L. Lebowitz 1975. Journal of Computational Physics 17:10-18

Caturla, M.J., N. Soneda, T. Diaz de la Rubia, M. Fluss, and M. Jaraíz 2006. Kinetic Monte Carlo simulations applied to irradiated materials: The effect of cascade damage in defect nucleation and growth. Journal of Nuclear Materials 351:78-87

Ceperley, D. M. and B. J. Alder 1980. Physical Review Letters: 45:566-569

Gilmer, G.H. and P. Bennema. 1972. Simulation of Crystal Growth With Surface Diffusion. Journal of Applied Physics 43:1347-1360

Gilmer, G.H. 2007. Private communication

Gubernatis, J. E. 2003. The Monte Carlo Method in the Physical Sciences, In AIP Conference. Proceedings, ed. James E. Gubernatis, 690

Huang, H., G. H. Gilmer, and T. Diaz de la Rubia. 1998. An atomistic simulator for thin film deposition in three dimensions, Journal of Applied Physics 84: 3636-3649.

Kadau, K., C. Rosenblatt, J. L. Barber, T. C. Germann, Z. Huang, P. Carlès, and B. J. Alder. 2007. The importance of fluctuations in fluid mixing. Proceedings of the National Academy of Sciences. 104:7741-7745

Kalos, M. H. 1962. Physical Review: 128:1791-1795

Kalos, M. H., D. Levesque and L. Verlet 1974. Physical Review A9:2178-2195

Kalos, M. H. and P. A. Whitlock 1986. Monte Carlo Calculations: Wiley

Kalos, M. H. and F. Pederiva 2000. Physical Review Letters 85:3547-3551

Landau, D. P. and K. Binder 2000: A Guide to Monte Carlo Simulations in Statistical Physics: Cambridge University Press

Landau, D. P. 2003. The Metropolis Monte Carlo Method in Statistical Physics. In The Monte Carlo Method in the Physical Sciences, AIP Conference Proceedings, ed. James E. Gubernatis, 690: 134-146

Law, M. E., G. H. Gilmer, and M. Jaraíz 2000. Simulation of Defects and Diffusion Phenomena in Silicon, MRS Bulletin 45-50, June

McMillan, W. L. 1965. Physical Review A138: 442-445

MCNP 2007. Available via <http://mcnp-green .lanl.gov/index.html>

Metropolis, N., A. Rosenbluth, M. Rosenbluth, A. Teller, and E. Teller 1953. Journal of Chemical Physics. 21:1087-1092

Oppelstrup, T., V. V. Bulatov, G. H. Gilmer, M. H. Kalos, and B. Sadigh 2006. First Passage Monte Carlo Algorithm: Diffusion without All the Hops. Physical Review Letters 97:230602-230605

Pandaripande, V. 2003. Quantum Monte Carlo Simulations of Nuclei and Nuclear Reactions. In The Monte Carlo Method in the Physical Sciences, AIP Conference Proceedings, ed. James E. Gubernatis, 690:261268 
Pauli, W. 1927. Zeitschrift für Physik.43:601-623

Pieper, S.C. 2007. Private communication

Quigg, C. 1983. Theories of Strong, Weak, and Electromagnetic Interactions: Benjamin-Cummings

Schmidt, K. E., P. Niyaz, A. Vaught, and M. A. Lee 2005.Physical Review E71, 016707

Sweezy, J., F. Brown, T. Booth, J. Chiaramonte, and B. Preeg 2005. Radiation Protection Dosimetry 116: 508515

Umrigar, C. and J. Toulouse. 2007. Private communication Wilson, K. 1974. Physical Review D10: 2445-2459

\section{AUTHOR BIOGRAPHY}

Malvin H. Kalos is a physicist at the Lawrence Livermore National Laboratory. He received his $\mathrm{PhD}$ in Physics at the University of Illinois in 1952. After a year of postdoctoral research at Illinois, and two at Cornell he joined the staff of United Nuclear Corporation. In 1964, he became a Senior Research Scientist, later Research Professor and then Professor of Computer Science at the Courant Institute of Mathematical Sciences, New York University. In 1989, he returned to Cornell as Professor of Physics and director of the Theory Center. His research interests include Monte Carlo Methods and computational many-body physics, including particle transport, quantum Monte Carlo, and statistical physics. He is the recipient of the 1989 Feenberg Memorial Medal for advancement of many-body theories from first principles. 